\title{
Wie können Ergebnisse der Kompetenzdiagnostik in Forschungsprojekten sinnvoll zurückgemeldet werden?
}

\author{
Dominik Leiss • Katrin Rakoczy
}

Zusammenfassung: Rückmeldung von (Forschungs-)Ergebnissen an Lernende, Lehrende und institutionelle Akteure stellt ein zentrales Element im Spannungsfeld kompetenzdiagnostischer Forschungsarbeiten dar: Schülern/-innen sollen möglichst individuelle Rückmeldungen zu erbrachten Leistungen gegeben werden, Lehrkräfte sind am Lernstand der eigenen Klasse interessiert, Schulämter und Ministerien sind Informationen über Ergebnisse aktueller Forschungen anzubieten. Damit eine Gestaltung und Umsetzung von Rückmeldungen auf derart unterschiedlichen Ebenen erfolgreich gelingen kann, gilt es, sich verschiedener zentraler Fragen bewusst zu sein: 1) Wer ist Empfänger/-in der Rückmeldung? Was ist das Ziel der Rückmeldung? 2) Welche Ergebnisse sollen zurückgemeldet werden? 3) Wie sollte eine Rückmeldung aus theoretischer Sicht idealerweise gestaltet sein? Und 4) wie ist eine solche Rückmeldung von gegebenen Rahmenbedingungen abhängig? Eine Diskussion dieser Fragen soll im vorliegenden Beitrag erfolgen, eine abschließend überblicksartig aufgeführte „Checkliste für Projektergebnisrückmeldungen“ ist dabei als Angebot an Forschungsprojekte im Kontext kompetenzdiagnostischer Fragestellungen zu verstehen.

Schlüsselwörter: Kompetenzdiagnostik · Projektergebnisse $\cdot$ Rückmeldung

\section{How to report on results of competence assessment research?}

Abstract: Reporting on research results to students, teachers and institutions is a big challenge within competence assessment research: students individually have to be given feedback about how they performed, teachers have to be provided with information about their classes, education authorities are interested in actual research results. For feedback being helpful for students, teachers and institutions, the following questions have to be thought of: (1) Who is the feedback given to? What is the aim of the feedback? (2) Which results shall be reported? (3) How should feedback theoretically look like? And (4) how does feedback depend on the given general

(C) Springer Fachmedien Wiesbaden 2013

Prof. Dr. D. Leiss $(\bowtie)$

Institut für Mathematik und ihre Didaktik, Leuphana Universität Lüneburg,

Scharnhorststr. 1, 21335 Lüneburg, Deutschland

E-Mail: dominik.leiss@leuphana.de

Dr. K. Rakoczy $(\bowtie)$

Deutsches Institut für Internationale Pädagogische Forschung,

Schloßstr. 29, 60486 Frankfurt am Main, Deutschland

E-Mail: rakoczy@dipf.de 
framework? The given article discusses these questions and offers a "checklist" to research projects with a focus on competence assessment.

Keywords: Competence assessment $\cdot$ Feedback $\cdot$ Research results

Als im Bereich der Kompetenzdiagnostik Forschende/r ist man häufig mit der Situation konfrontiert, dass unterschiedliche Akteurinnen und Akteure des Bildungssystems über Ergebnisse eines Forschungsprojekts, an dem sie in irgendeiner Weise beteiligt waren, informiert werden möchten. Häufig ist es aufgrund verschiedener Rahmenbedingungen nicht möglich, die Rückmeldung an Beteiligte ausschließlich aufgrund theoretisch wünschenswerter Kriterien zu gestalten. Entsprechend müssen sich Forscher/innen zunächst folgende Fragen stellen und diese bei der Gestaltung der Rückmeldung in einer adäquaten Form beantworten:

1. An wen soll die Rückmeldung gegeben werden und welche Ziele könnte der Empfänger bzw. die Empfängerin damit verfolgen?

2. Welche Ergebnisse werden im Projekt erzeugt und eignen sich für die Rückmeldung an die Teilnehmenden?

3. Welche theoretischen Erkenntnisse über die (lernförderliche) Gestaltung von Rückmeldung liegen vor?

4. An welche organisatorischen Rahmenbedingungen ist die Erstellung bzw. Vergabe von Rückmeldung gebunden?

Die für die Ergebnisdarstellung getroffenen Entscheidungen (und die damit verbundenen Beschränkungen) gilt es so weit wie möglich auch mit den verschiedenen Akteuren und Akteurinnen offen zu kommunizieren, um diesen das Verständnis der Rückmeldung zu erleichtern. Welche Entscheidungen dies sein können, soll im Folgenden überblicksartig beschrieben werden.

\section{Empfängerinnen, Empfänger und ihre Ziele}

Zunächst gilt es, sich bewusst zu machen, wer der Empfänger bzw. die Empfängerin der zu gebenden Rückmeldungen ist, und zu berücksichtigen, dass die verschiedenen Akteure und Akteurinnen gänzlich unterschiedliche Verwendungszwecke bzw. Interessen mit den Rückmeldungen verfolgen. Dabei spielt ebenfalls eine Rolle, inwiefern der entsprechende Personenkreis aus Experten beziehungsweise Novizen auf dem jeweiligen Forschungsgebiet besteht und entsprechend auch unterschiedlich gut in der Lage ist, mit verschiedenen Darstellungsformen umzugehen. Folglich sind die Ergebnisse nicht nur hinsichtlich einer möglichst verständlichen und ansprechenden Informationsweitergabe, sondern auch inhaltlich differenziert für die verschiedenen Zielgruppen aufzuarbeiten. Wir unterscheiden im Folgenden zwischen Lernenden, Lehrpersonen und institutionellen Akteuren.

Es erscheint naheliegend, Lernenden als Gegenleistung für ihr Engagement im jeweiligen Forschungsprojekt personenbezogene Informationen rückzumelden. Inwiefern dies 
in Form einer Rückmeldung zu individuellen inhaltlichen Leistungen geschehen sollte, die den Lernbedarf aufzeigt, oder in Form eines Vergleichs mit einer Sozialnorm wie der eigenen Schulklasse, ist eine eigene Forschungsfrage (siehe unten). Einige Ergebnisse deuten allerdings darauf hin, dass eine individuelle Rückmeldung, die sich auf die Lernprozesse der Lernenden bezieht, in motivationaler und leistungsbezogener Hinsicht einer sozial vergleichenden Rückmeldung überlegen ist.

Bezüglich der Rückmeldung an Lehrpersonen gibt es wenig verallgemeinerbare Erfahrungen (vgl. Kohler und Schrader 2004). Konsens besteht darin, dass es für Lehrpersonen hilfreich ist, die Leistung ihrer Lerngruppe im Vergleich zur relevanten Bezugsgruppe oder in Bezug auf eine kriteriale Bezugsnorm wie zum Beispiel die Curricula der Länder oder die nationalen Bildungsstandards zu sehen. Alleiniges Bereitstellen von Informationen hat nach Kohler und Schrader (2004) bisher nicht zu Verbesserungen geführt (vgl. auch Nachtigall und Jantowski 2007). Die Ergebnisse von Kompetenzmessungen könnten jedoch - bei entsprechender Aufbereitung - von Lehrkräften als diagnostisches Tool genutzt werden und somit im Prozess der Optimierung pädagogischen Handelns einen wichtigen Beitrag leisten. Ein gelungenes Beispiel hierfür stellen die didaktischen Kommentare zu den Leistungsrückmeldungen aus Vergleichsarbeiten (VERA) dar (vgl. Pant 2013 in diesem Heft). Hier werden nicht bloß quantitative Ergebnisse präsentiert, sondern sowohl die Ergebnisse als auch die eingesetzten Testmaterialien werden einer didaktischen Analyse unterzogen, wodurch den Lehrpersonen ermöglicht wird, die eigenen Klassenleistungen inhaltlich zu reflektieren und Anregungen für den Unterricht zu gestalten. Diese Art der Aufbereitung ist zwar mit einem erheblichen Mehraufwand für das jeweilige Projekt verbunden, wenn man aber nicht nur im Sinne einer Belohnung der Lehrkräfte diese informieren, sondern auch einen inhaltlichen Einfluss auf deren Lehrexpertise beziehungsweise Unterrichtsgestaltung erreichen möchte, dann bedarf es eines differenzierteren Feedbacks (vgl. Maier 2008).

Anders als bei Rückmeldungen an die unmittelbar am Lehr-Lernprozess Beteiligten erscheint es für institutionelle Akteure wie Schulämter und Ministerien notwendig, dass gewonnene Ergebnisse auf einem angemessenen Aggregationsniveau dargestellt werden. Dabei gilt es, sich als Forscher/-in bewusst zu machen, dass insbesondere diese Akteure ein Interesse an mehr oder weniger stark inhaltlich orientiertem Steuerungswissen haben. Entsprechend sollte einer bloßen Outputkontrolle mit weitreichenden Sanktionen (wie z. B. bei den Schulleistungstests im Rahmen des amerikanischen „No child left behind“Gesetzes) entgegengewirkt werden. Vielmehr sollten im Rahmen eines „Formativen Systemmonitorings“" Möglichkeiten aufgezeigt werden, wie Lehr-Lerninhalte auf Systemebene erfasst und gegebenenfalls zur Modifikation von Curricula verwendet werden können.

\section{Projektergebnisse}

So wünschenswert es erscheint, allen in Abschn. 1 angeführten Akteuren und Akteurinnen Informationen über die Analysen der jeweiligen Forschungsvorhaben zukommen zu lassen, so stark steht und fällt dieser Wunsch mit der Art der erzielten Ergebnisse bzw. der im Projekt verfolgten Forschungsfragen. So bestimmt eine Reihe von Faktoren, ob, wem 
und auf welche Art und Weise Ergebnisse rückgemeldet werden können bzw. sollten. Die folgenden Fragen sollen einen exemplarischen Eindruck hiervon vermitteln:

- Wie anwendungsorientiert ist die Forschung? Ermöglichen die Ergebnisse handlungsleitende Folgerungen oder handelt es sich eher um Grundlagenforschung?

- Auf welcher Ebene liegen die Ergebnisse? Wurden zum Beispiel kognitive Lernprozesse auf individueller Ebene analysiert oder können die Ergebnisse auf einer schulischen Makroebene verortet werden?

- Welche Stichprobe wurde untersucht? Wurden die Ergebnisse im Rahmen einer qualitativen Fallstudie erhoben oder wurde eine repräsentative Stichprobe untersucht?

- Wie ,gut“ sind die Ergebnisse? Handelt es sich um positive Ergebnisse oder sind diese eher kompromittierend? Zu welchem Kriterium können sie in Beziehung gesetzt werden (z. B. Klasse, individueller Verlauf etc.)?

\section{Theoretische Erkenntnisse über die Gestaltung von Leistungsrückmeldung}

Aus theoretischer Sicht ist es wünschenswert, Rückmeldung über Leistungsergebnisse so zu gestalten, dass sie im Sinne der aus zahlreichen Studien gewonnenen theoretischen und empirischen Erkenntnisse motivations- und lernförderlich ist. Da dies mit erheblichem Aufwand verbunden ist, wird es zumeist nur dann umfassend realisiert, wenn die Rückmeldung selbst Forschungsgegenstand ist und sie einen laufenden Lehr-Lern-Prozess in positiver Weise beeinflussen soll. Studien, die sich mit Rückmeldung in einem laufenden Lehr-Lern-Prozess als Forschungsgegenstand beschäftigen und somit primär Lernende als Proband/-innen untersuchen, haben folgende Kriterien für die Gestaltung der Rückmeldung hervorgebracht:

Nach Hattie und Timperley (2007) sollte eine lernförderliche Rückmeldung Informationen beinhalten, wie die Distanz zwischen einer tatsächlich erzielten Leistung und einem gewünschten Lernziel verringert werden kann. Diese Art von Information ist auf der Aufgabenebene lokalisiert. Rückmeldung auf der Verarbeitungsebene, d.h. Rückmeldung, die hilft, Fehler zu erkennen, Informationen zu suchen und Strategien zu verwenden, ist darüber hinaus hilfreich, wenn es um komplexe Aufgaben geht, für deren Lösung tiefergehendes Lernen notwendig ist (vgl. Hattie und Timperley 2007). Kluger und DeNisi (1996) finden ebenfalls, dass Rückmeldung, die sich direkt auf bearbeitete Aufgaben bezieht (Aufgaben- oder Verarbeitungsebene), wirksamer ist als Rückmeldung, die sich auf die Leistung insgesamt und damit auf die Person des/der Lernenden bezieht (Selbstebene). Dadurch, dass Rückmeldung auf die einzelnen bearbeiteten Aufgaben und damit verbundene Lernprozesse und nicht auf den Gesamterfolg in einem Test fokussiert, soll die Leistung mit Anstrengung und nicht mit Fähigkeit assoziiert werden (siehe zu Kausalattributionen McElvany und Rjosk 2013 in diesem Heft). Die Rückmeldung soll die Verbindung zwischen Anstrengung und Erfolg aufzeigen, damit die Anstrengungsbereitschaft der Lernenden aufrechterhalten bleibt beziehungsweise erhöht wird (vgl. Mory 1996).

Im Rahmen der Selbstbestimmungstheorie von Deci und Ryan (vgl. z. B. Ryan und Deci 2002) wird davon ausgegangen, dass Rückmeldung den Lernprozess dann positiv beeinflusst, wenn sie durch Information über die individuellen Kompetenzen der 
Lernenden deren grundlegendes Bedürfnis nach Kompetenz unterstützt (vgl. z. B. Deci et al. 1999). Das bedeutet, die Rückmeldung sollte den Lernenden zeigen, dass ihnen etwas zugetraut wird, welche Fortschritte sie bereits gemacht haben, wo sie sich noch verbessern können etc. Dagegen gilt Feedback, welches den Lernenden aufzeigt, wie sie sich (hätten) verhalten sollen, als motivationshinderlich, da es eine Bedrohung des Bedürfnisses nach Autonomie darstellt. Damit ist jedoch nicht gemeint, dass die richtige Lösung einer Aufgabe genannt wird, was durchaus als lernförderlich eingeschätzt wird (vgl. Sansone 1986), sondern dass betont wird, was von dem/der Lernenden erwartet wird bzw. wurde, um Druck zu erzeugen, eine bestimmte Leistung zu erbringen.

Darüber hinaus sollte Rückmeldung in möglichst enger zeitlicher Nähe zu dem Leistungsergebnis erfolgen. Außerdem ist es wichtig, dass die Rückmeldung persönlich relevant ist. Sie sollte aufzeigen, wozu die Lernenden die Rückmeldung gebrauchen beziehungsweise bei welcher Gelegenheit sie das Gelernte anwenden können.

Rückmeldung wirkt sich nicht auf alle Lernenden gleich aus, sondern sie wirkt in Abhängigkeit von bestimmten Merkmalen der Lernenden und der Situation. Häufig wird das Leistungsniveau der Lernenden als Bedingung für die Wirksamkeit von Feedback thematisiert. Nach Sansone (1986) bestimmt die Leistung den Einfluss von sozial vergleichendem Feedback dahingehend, dass die leistungsstärkeren Lernenden stärker von solchem Feedback profitieren. Laut Kluger und DeNisi (1996) sowie Butler und Winne (1995) bestimmt auch die Zielorientierung die Wirkung von Feedback. Es wird davon ausgegangen, dass sich Rückmeldung, die eine individuelle Bezugsnorm anlegt, sich besonders positiv bei Schülern/-innen mit Lernzielorientierung auswirkt. Dagegen sollte sozial vergleichende Rückmeldung oder Rückmeldung, die eine kriteriale Bezugsnorm beinhaltet, vor allem bei Schülerinnen und Schülern positiv wirken, die eine Leistungszielorientierung aufweisen.

\section{Organisatorische Rahmenbedingungen}

Möchte man den Beteiligten an einer Studie Rückmeldung über die Ergebnisse geben, sind die in Abschn. 3 genannten Kriterien zur Gestaltung lernförderlicher Rückmeldung aufgrund der spezifisch gegebenen organisatorischen Rahmenbedingungen häufig nicht zu erfüllen. Darunter fallen insbesondere die Begrenztheit der zur Verfügung stehenden zeitlichen und monetären Ressourcen sowie die vorliegenden Datenschutzbestimmungen.

Aufgrund begrenzter Ressourcen ist das Kriterium enger zeitlicher Nähe häufig nicht zu realisieren, da die Kodierung der Aufgabenbearbeitungen und die Aufbereitung der Daten mit erheblichem Aufwand verbunden sind. Dieses Problem kann jedoch dadurch reduziert werden, dass den Adressaten zusammen mit den Ergebnissen die zuvor bearbeiteten Aufgaben zur Verfügung gestellt werden.

Eine Rückmeldung, die detailliert auf die einzelnen Aufgabenbearbeitungen und die damit verbundenen Lernprozesse eingeht, um die Distanz zum Lernziel zu verringern, ist ebenfalls mit sehr großem Aufwand verbunden. Auch damit die Rückmeldung persönlich relevante Informationen enthält und die Lernenden in ihren Bedürfnissen nach Kompetenz und Autonomie unterstützt, ist eine intensive Auseinandersetzung mit den einzelnen Schülerlösungen und Lösungswegen notwendig, die in großen Stichproben 
nicht realisierbar ist. Im Falle knapper Ressourcen bei großen Stichproben könnte auf eine Einstufung der Lernerfähigkeiten in einem Kompetenzniveaumodell als Alternative zurückgegriffen werden. In einem solchen Modell sollte möglichst praxisnah beschrieben werden, über welche Operationen und Prozesse Lernende auf bestimmten Niveaus verfügen. Durch die Verortung auf der Kompetenzniveauskala sehen die Lernenden, welche Prozesse schon beherrscht werden und welche Prozesse auf den höheren Niveaus noch zu erreichen sind.

Generell ist ein sensibler Umgang mit der Rückmeldung von Lernergebnissen unerlässlich. So sollte, wann immer möglich, ein persönliches Gespräch mit den Adressaten stattfinden, um die Ergebnisse zu erklären und die Möglichkeit zu geben, Rückfragen zu stellen. Wissenschaftler/-innen, Lernende, Lehrpersonen und institutionelle Akteure verwenden in der Regel sehr unterschiedliche Sprachen, um Leistungsergebnisse zu beschreiben und zu interpretieren. Um sich gegenseitig zu verständigen, bedarf es einer ausführlichen und einfühlsamen Kommunikation. Sie sollte sowohl das Bemühen um eine verständliche und ansprechende Darstellung der Ergebnisse als auch die Fort- und Weiterbildung der Akteurinnen und Akteure zum Umgang mit Forschungsergebnissen beinhalten.

\section{Checkliste für die Projektergebnisrückmeldung}

Im Folgenden werden zentrale Kriterien für die Gestaltung von Ergebnisrückmeldungen aus Kompetenzmessungen in Form einer Checkliste zusammengefasst.

\section{Empfänger/innen und ihre Ziele:}

- Sind die Rückmeldungen bezüglich (Fach-)Sprache, grafischen Darstellungen und allgemeinem Layout so aufbereitet, dass sie von der jeweiligen Zielgruppe verstanden werden können?

- Sind die Rückmeldungen inhaltlich so aufbereitet, dass die enthaltenen Informationen von den Empfängern/innen als interessant und hilfreich in ihrer weiteren Arbeit angesehen werden?

- Ist das Aggregationsniveau der Rückmeldungen für den/die Empfänger/in angemessen?

Projektergebnisse:

- Eignen sich die erzielten Ergebnisse für eine Rückmeldung, die über das bloße Informieren der Beteiligten auf einem allgemeinem Niveau hinausgeht?

- Werden durch die dargelegten Inhalte die Persönlichkeitsrechte gewahrt oder handelt es sich eventuell sogar um nicht veröffentlichbare Ergebnisse?

- Wurden die Daten untereinander in Beziehung gesetzt?

- Ist die „Unsicherheit“ von Kompetenzdiagnostik bzw. die Relativierung der Absolutheit von Kompetenzzuschreibungen als Einschränkung für die Interpretation der Ergebnisse transparent?

Theoretische Erkenntnisse:

- Hilft die Rückmeldung der Zielperson, die Distanz zwischen ihrer Leistung und dem Lernziel zu verringern? 
- Unterstützt die Rückmeldung die Zielperson in ihren Bedürfnissen nach Kompetenz und Autonomie?

- Erfolgt die Rückmeldung in zeitlicher Nähe zur Leistungserfassung?

- Enthält die Rückmeldung persönlich relevante Informationen für die Zielperson?

Organisatorische Rahmenbedingungen:

- Welche Ressourcen stehen für die Rückmeldung zur Verfügung?

- Wie sind die Datenschutzbestimmungen?

- Besteht außer der schriftlichen Rückmeldung auch die Möglichkeit, ein gemeinsames Treffen zu organisieren, bei dem die Projektergebnisse besprochen werden?

Danksagung: Diese Veröffentlichung wurde ermöglicht durch Sachbeihilfen der Deutschen Forschungsgemeinschaft (Kennz.: KL 1057/10-2 und BL 275/17-1) im Schwerpunktprogramm „Kompetenzmodelle zur Erfassung individueller Lernergebnisse und zur Bilanzierung von Bildungsprozessen“ (SPP 1293).

\section{Literatur}

Butler, D. L., \& Winne, P. H. (1995). Feedback and self-regulated learning: A theoretical synthesis. Review of Educational Research, 65, 245-281.

Deci, E. L., Koestner, R., \& Ryan, R. M. (1999). A meta-analytic review of experiments examining the effects of extrinsic rewards on intrinsic motivation. Psychological Bulletin, 125, 627-668.

Hattie, J., \& Timperley, H. (2007). The power of feedback. Review of Educational Research, 77, $81-112$.

Kluger, A. N., \& DeNisi, A. (1996). The effects of feedback interventions on performance. A historical review, a meta-analysis, and a preliminary feedback intervention theory. Psychological Bulletin, 119, 254-284.

Kohler, B., \& Schrader, F.-W. (2004). Ergebnisrückmeldung und Rezeption. Von der externen Evaluation zur Entwicklung von Schule und Unterricht (Themenheft der Empirischen Pädagogik, Vol. 18(1)). Landau : VEP.

Maier, U. (2008). Rezeption und Nutzung von Vergleichsarbeiten - Ergebnisse einer Lehrerbefragung in Baden-Württemberg. Zeitschrift für Pädagogik, 11, 453-474.

McElvany, N., \& Rjosk, C. (2013). Wann kann Kompetenzdiagnostik negative Auswirkungen haben? In D. Leutner, E. Klieme, J. Fleischer \& H. Kuper (Hrsg.), Kompetenzmodelle zur Erfassung individueller Lernergebnisse und zur Bilanzierung von Bildungsprozessen: aktuelle Diskurse im DFG-Schwerpunktprogramm (18. Sonderheft der Zeitschrift für Erziehungswissenschaft, DOI: 10.1007/s11618-013-0387-z). Wiesbaden: VS Verlag für Sozialwissenschaften.

Mory, E. H. (1996). Feedback research. In D. H. Jonassen (Hrsg.), Handbook of research for educational communications and technology (S.919-956). New York: Macmillan.

Nachtigall, C., \& Jantowski, A. (2007). Die Thüringer Kompetenztests unter besonderer Berücksichtigung der Evaluationsergebnisse zum Rezeptionsverhalten. Empirische Pädagogik, 21, 401-410.

Pant, H. A. (2013). Wer hat einen Nutzen von Kompetenzmodellen? In D. Leutner, E. Klieme, J. Fleischer \& H. Kuper (Hrsg.), Kompetenzmodelle zur Erfassung individueller Lernergebnisse und zur Bilanzierung von Bildungsprozessen: aktuelle Diskurse im DFG-Schwerpunktprogramm (18. Sonderheft der Zeitschrift für Erziehungswissenschaft, DOI: 10.1007/s11618-0130388-y). Wiesbaden: VS Verlag für Sozialwissenschaften. 
Ryan, R. M., \& Deci, E. L. (2002). An overview of self-determination theory: An organismicdialectical perspective. In E. L. Deci \& R. M. Ryan (Hrsg.), Handbook of self-determination research (S.3-33). Rochester: University of Rochester Press.

Sansone, C. (1986). A question of competence: The effects of competence and task feedback on intrinsic interest. Journal of Personality and Social Psychology, 51, 918-931. 